\title{
Efeitos de diferentes frequências da estimulação elétrica nervosa transcutânea em relação à acomodação e à agradabilidade
}

\author{
Effects of different transcutaneous electrical nerve stimulation frequencies regarding \\ adaptation and pleasantness
}

\author{
Danilo de Oliveira Silva ${ }^{1}$, Amanda Schenatto Ferreira ${ }^{2}$, Ana Valéria Gonçalves², Marina Dalla Costa², Marina Zilio², \\ Andersom Ricardo Fréz ${ }^{3}$, Gladson Ricardo Flor Bertolini ${ }^{4}$ \\ ${ }^{1}$ Mestrando do Programa de Pós Graduação em Fisioterapia da Universidade Estadual Paulista "Júlio de Mesquita Filho" (UNESP), Presidente Prudente, SP; ${ }^{2}$ Discente \\ de Graduação em Fisioterapia da Universidade Estadual do Oeste do Paraná (UNIOESTE), Cascavel, PR; ${ }^{3}$ Mestre em Saúde, Interdisciplinaridade e Reabilitação pela \\ Universidade Estadual de Campinas (UNICAMP), Docente da graduação em Fisioterapia da Universidade Estadual do Centro Oeste do Paraná (UNICENTRO), Guarapuava, \\ PR; ${ }^{4}$ Doutor em Ciências da Saúde Aplicadas ao Aparelho Locomotor pela Faculdade de Medicina de Ribeirão Preto da Universidade de São Paulo (USP), Docente da \\ graduação em Fisioterapia e mestrado em Biociências e Saúde da UNIOESTE, Cascavel, PR.
}

\section{RESUMO}

Objetivos: Investigar os efeitos de diferentes frequências da estimulação elétrica nervosa transcutânea (TENS - transcutaneous electrical nerve stimulation), em relação à acomodação e agradabilidade nas diversas formas de aplicação.

Métodos: A amostra foi composta por 20 indivíduos, divididos aleatoriamente em três grupos, que foram submetidos à TENS, com três frequências diferentes: $7 \mathrm{~Hz}, 100 \mathrm{~Hz}$ e $255 \mathrm{~Hz}$, com duração de pulso de $250 \mu$ s, por 20 minutos, uma vez por semana no período de três semanas. Os voluntários eram orientados a indicarem o momento da primeira acomodação e as acomodações subsequentes. No final do período de coleta de dados, os indivíduos foram interrogados sobre a frequência que julgassem mais agradável.

Resultados: Para o limiar de acomodação, considerando todas as frequências analisadas, verificou-se que não houve diferença significativa $(\mathrm{p}>0,05)$, fato que também se repetiu para o número de acomodações em 20 minutos $(\mathrm{p}>0,05)$. Com relação à agradabilidade, 11 indivíduos indicaram a frequência de $7 \mathrm{~Hz}$ como a mais agradável.

Conclusões: Não houve diferença significativa no limiar de acomodação e no número de acomodações entre as frequências utilizadas. A frequência de $7 \mathrm{~Hz}$ foi apontada como a mais agradável seguida da frequência de $100 \mathrm{~Hz}$.

DESCRITORES: ESTIMULAÇÃO ELÉTRICA NERVOSA TRANSCUTÂNEA; PERCEPÇÃO DO TATO; MODALIDADES DE FISIOTERAPIA.

\section{ABSTRACT}

Aims: The aim of this study was to investigate the effects of different frequencies of transcutaneous electrical nerve stimulation (TENS) regarding to accommodation and pleasantness of the different forms of application.

Methods: The sample consisted of 20 subjects randomly divided into 3 groups, in which they were submitted to 3 different frequencies of TENS: $7 \mathrm{~Hz}, 100 \mathrm{~Hz}, 255 \mathrm{~Hz}$, pulse duration of $250 \mu \mathrm{s}$, for 20 minutes once a week over three weeks. The volunteers were instructed to indicate the first and subsequent accommodation moments. At the end of the period of data collection, subjects were asked about which frequency they thought most enjoyable.

Results: For the threshold of adaptation, considering all frequencies analyzed, it was found that there was no significant difference ( $p>0.05$ ), a fact that was also repeated for the number of adaptations in 20 minutes ( $>00.05)$. With regard to pleasantness, 11 subjects indicated the frequency of $7 \mathrm{~Hz}$ as the most enjoyable.

Conclusions: There was no significant difference in threshold for adaptation and the number of adaptations between the frequencies used. The frequency of $7 \mathrm{~Hz}$ was identified as the most enjoyable then the frequency of $100 \mathrm{~Hz}$.

KEY WORDS: TRANSCUTANEOUS ELECTRICAL NERVE STIMULATION; PERCEPTION OF TOUCH; PHYSICAL THERAPY MODALITIES. 


\section{INTRODUÇÃO}

A estimulação elétrica nervosa transcutânea (TENS - transcutaneous electrical nerve stimulation) é uma técnica analgésica não-invasiva usada para o manejo sintomático de dor aguda e crônica. ${ }^{1}$ É gerada por um aparelho de corrente de baixa frequência do tipo bidirecional e assimétrico que pode variar de 1 a $250 \mathrm{~Hz}$, não provocando ionização. ${ }^{2}$ A TENS é aplicada em uma variedade de frequências, intensidades e durações de pulso, sendo que as mais utilizadas são: alta frequência (convencional), baixa frequência (acupuntura), em rajadas (burst) e breve intensa. ${ }^{3,4}$

As modalidades, relacionadas com as frequências da corrente, estão diretamente implicadas no mecanismo de ação. A de alta frequência age ativando a modulação da dor no nível da medula espinhal, ou seja, tem ação mais evidente com estimulação sensorial. Por outro lado, a forma acupuntura atua em nível motor, produzindo a liberação de opioides endógenos, que bloqueiam os sinais nociceptivos, exercendo, portanto, ação analgésica. ${ }^{5}$

Um dos assuntos abordados na literatura acerca da TENS é a acomodação neural. O processo de acomodação ocorre quando uma resposta fisiológica é diminuída continuamente, com a aplicação repetitiva de um mesmo estímulo. Como resposta inicial, os receptores agem com alta frequência de impulsos, ao passo que quando o estímulo torna-se constante, a frequência de impulsos diminui progressivamente. A manutenção da frequência do estímulo é fundamental para o aparecimento da acomodação. Com relação à agradabilidade sabese que os sujeitos sentem diferentes frequências de correntes terapêuticas de formas distintas. Portanto, a sensação mesmo que subjetiva de cada indivíduo deve ser levada em consideração, no momento da escolha da frequência, deve-se buscar o melhor equilíbrio entre resultado e o mínimo de desconforto para cada indivíduo. ${ }^{6,7}$

Apesar de já existir um estudo que aborda diferentes frequências da TENS, ${ }^{8}$ há uma lacuna acerca de como as diferentes frequências atuam no processo de acomodação. Dessa forma, os valores de frequência e duração de pulso são discrepantes na literatura, não existindo um consenso sobre qual a melhor frequência ou sobre como cada frequência pode se comportar, o que dificulta e reduz a qualidade de intervenções. Sendo assim, o objetivo deste estudo foi investigar os efeitos de diferentes frequências da TENS $(7 \mathrm{~Hz}$, $100 \mathrm{~Hz}$ e $255 \mathrm{~Hz}$ ) com relação à acomodação e à agradabilidade.

\section{MÉTODOS}

Este estudo caracterizou-se como um ensaio clínico controlado, cruzado, aleatório, com caráter quantitativo e amostragem por conveniência. O estudo foi aprovado pelo Comitê de Ética em Pesquisa Envolvendo Seres Humanos da Universidade Estadual do Oeste do Paraná (UNIOESTE), parecer n 115/2013. A coleta de dados foi realizada na Clínica-Escola de Fisioterapia da Universidade Estadual do Oeste do Paraná (UNIOESTE), campus Cascavel. Todos os participantes assinaram um Termo de Consentimento Livre e Esclarecido (TCLE).

\section{Amostra e procedimentos}

Após o esclarecimento dos objetivos e procedimentos do estudo, os voluntários foram submetidos à triagem para o registro de dados e identificação de possíveis fatores de exclusão. Como critérios de inclusão, os voluntários deveriam ter disponibilidade para participar das avaliações e testes nos dias e horários pré-determinados e apresentar integridade sensitiva dos dermátomos $\mathrm{C} 6$ a $\mathrm{C} 8$. Os critérios de exclusão foram: contraindicação para o uso de qualquer tipo de eletroestimulação; indivíduos que faziam uso de marcapasso ou de qualquer implante metálico; estado febril; neoplasia; tuberculose; déficit cognitivo ou sensorial; e suspeita ou diagnóstico de trombose venosa profunda. Depois de constatada a elegibilidade para o estudo, os voluntários recrutados assinaram o TCLE.

O aparelho de TENS utilizado foi o Physiotonus Four (BIOSET ${ }^{\circledR}$, Rio Claro, Brasil). Os eletrodos foram colocados no membro superior dominante, sendo um disposto na região entre o epicôndilo medial e olécrano (sulco do nervo ulnar) e o outro medial à inserção do bíceps braquial (região do nervo mediano), no trajeto dos nervos em regiões nas quais eles estão mais superficiais. Após a assepsia do local, os eletrodos, com $8 \mathrm{~cm}^{2}$ cada, foram fixados à pele com fita adesiva, sendo utilizado gel condutor entre o eletrodo e a pele.

A estimulação foi realizada durante 20 minutos, uma vez por semana, com duração de pulso fixa de $250 \mu \mathrm{s}$, durante períodos de uma semana que se alternavam em três frequências diferentes: $7 \mathrm{~Hz}$, com intensidade mínima forte, porém agradável; 100 $\mathrm{Hz}$, frequência comumente utilizada para analgesia via comportas; e, $255 \mathrm{~Hz}$, a máxima frequência disponível no equipamento. Os voluntários foram divididos em três grupos alocados de maneira aleatória em cada frequência e, após uma semana, um rodízio 
era realizado e os sujeitos eram submetidos a outro tipo de frequência. Ao final do experimento, todos os voluntários foram submetidos às três diferentes frequências de estimulação.

\section{Avaliação da acomodação}

Utilizando-se um cronômetro, foi registrado o tempo exato em que os voluntários sentiram uma redução na intensidade de estímulo inicial (limiar de acomodação). Para isso, os voluntários foram orientados a relatar ao pesquisador o momento em que sentiam essa redução. Nesse momento o avaliador aumentava a intensidade do estímulo até que o voluntário relatasse a mesma intensidade inicial. Este procedimento foi executado cada vez que o indivíduo sentiu a diminuição da intensidade, registrando o número de acomodações relatadas durante o tempo da aplicação.

\section{Avaliação da agradabilidade}

Após o término de todas as aplicações, foi entregue um questionário aos voluntários, no qual deveriam indicar em qual das três semanas de intervenção a estimulação foi mais agradável.

\section{Análise estatística}

A análise da normalidade foi realizada por meio do teste de Kolmogorov-Smirnov, e os dados do limiar e número de acomodações foram analisados com o teste de Friedman. Para o tamanho de amostra obtido, visando avaliar o limiar de acomodação, observandose como diferença mínima entre as médias do tempo da primeira acomodação, 145 segundos, bem como desvio padrão de 150, com três grupos amostrais e nível de significância de $5 \%$, observou-se poder do teste de $80 \%$. Na comparação da agradabilidade, foi utilizado o Qui-quadrado de Pearson. Em todos os casos o nível de significância aceito foi de $5 \%$.

\section{RESULTADOS}

Inicialmente foram recrutados 30 indivíduos, no entanto, dez não compareceram no dia da coleta. A amostra ficou então definida em 20 indivíduos saudáveis, sendo seis mulheres e 14 homens, com idade média de 20,2 $\pm 3,07$ anos, massa corporal 74,5 $\pm 19,7 \mathrm{~kg}$, estatura $1,72 \pm 0,87 \mathrm{~m}$ e índice de massa corporal $25,1 \pm 4,95 \mathrm{~kg} / \mathrm{m}^{2}$. Os voluntários foram divididos em três grupos e alocados de maneira aleatória para cada frequência, conforme descrito em Métodos.

Verificou-se que não houve diferença significativa no tempo da primeira acomodação (limiar de acomodação) entre as três frequências analisadas, fato que também se repetiu para o número de acomodações ao longo dos 20 minutos (Tabela 1).

Em relação à agradabilidade, houve diferença significativa entre as frequências utilizadas $(p=0,002)$, sendo que as frequências mais baixas foram em maior número referidas como mais agradáveis (Tabela 2).

Tabela 1. Tempo da primeira acomodação e o número de acomodações das diferentes frequências da estimulação elétrica nervosa transcutânea (TENS), em um período de 20 minutos de aplicação.

\begin{tabular}{|c|c|c|c|c|c|c|}
\hline \multirow{2}{*}{ Frequência $(\mathrm{Hz})$} & \multicolumn{3}{|c|}{$\begin{array}{l}\text { Limiar de acomodação* } \\
\text { (em segundos) }\end{array}$} & \multicolumn{3}{|c|}{ Número total de acomodações } \\
\hline & Q1 & Mediana & Q3 & Q1 & Mediana & Q3 \\
\hline 7 & 135,5 & 228,5 & 312,5 & 2,0 & 2,5 & 5,0 \\
\hline 100 & 100,0 & 208,5 & 285,0 & 1,0 & 2,0 & 3,8 \\
\hline 255 & 111,0 & 120,0 & 240,0 & 1,0 & 2,0 & 3,8 \\
\hline
\end{tabular}

Q1: quartil 25; Q3: quartil 75.

* Para o limiar de acomodação, o padrão de medida foi em segundos.

Valores de $p$ encontrados para o teste de Friedman: limiar de acomodação $p=0,848$ e número total de acomodações $p=0,154$.

Tabela 2. Preferência dos indivíduos em relação às diferentes frequências utilizadas na estimulação elétrica nervosa transcutânea (TENS).

\begin{tabular}{ccc} 
Frequência $(\mathbf{H z})$ & $\begin{array}{c}\text { Número de indivíduos que referiram ser } \\
\text { a mais agradável }\end{array}$ & $\mathbf{p}^{*}$ \\
\hline 7 & 11 & \\
100 & 8 & 0,002 \\
255 & 1 & \\
\hline
\end{tabular}




\section{DISCUSSÃO}

Entre os vários procedimentos não farmacológicos existentes para o tratamento sintomático da dor crônica e aguda, a TENS tem se destacado como um excelente recurso terapêutico, amplamente utilizado pelos profissionais da área da saúde. ${ }^{9}$ A TENS mostra-se eficaz em diferentes aplicações, como no alívio da dor durante o trabalho de parto; para alívio imediato da dor crônica em pacientes com disfunção temporomandibular do tipo miofascial; e no controle da dor pós-operatória de cirurgia de revascularização do miocárdio, evitando a utilização excessiva de analgésicos, além de melhorar a força muscular respiratória, principalmente na força expiratória máxima. ${ }^{10-12} \mathrm{~A}$ acomodação neural, definida como a diminuição da percepção do estímulo, é descrita como um fator inconveniente na aplicação da TENS. Devido a isso, são necessárias pesquisas que busquem formas de amenizar tal problema. ${ }^{13}$

A carência de parâmetros para o uso de correntes eletroterapêuticas é um importante viés clínico, sendo utilizado o empirismo como base terapêutica, inclusive para evitar a acomodação, o que também foi evidenciado com a TENS. ${ }^{6,9}$ No presente estudo, não houve diferença significativa entre as frequências com relação à acomodação, demostrando que não existe uma frequência ótima para evitar esse fator dentro dos parâmetros utilizados. Uma forma de minimizar essa ocorrência é ajustar frequentemente a amplitude da corrente. ${ }^{14}$

Raimundo et al., ${ }^{15}$ ao avaliar o nível de serotonina sistêmica após a aplicação de corrente TENS de baixa $(4 \mathrm{~Hz})$ e alta frequência $(100 \mathrm{~Hz})$, obtiveram que frequências mais altas demonstraram maior liberação de serotonina em relação às mais baixas, e sendo esta uma substância relacionada ao bem-estar, infere-se que frequências mais altas seriam mais agradáveis. Em razão disto, o mesmo estudo cita ainda que nas correntes de baixa frequência, a sensação de abalos musculares pode ter inibido a liberação de serotonina, o que não ocorreu nas altas frequências, pelo fato de atuarem apenas em nível sensorial, justificando assim os resultados obtidos..$^{15}$ Esses achados são discordantes em relação à avaliação da agradabilidade encontrada no presente estudo, em que um maior número de indivíduos julgaram as frequências mais baixas como as mais agradáveis. Salienta-se que no presente estudo, além do uso das frequências próximas às citadas, utilizou-se $255 \mathrm{~Hz}$, pelo fato de ser a máxima frequência limite do equipamento. A utilização dessa frequência muito alta pode ter criado um viés em relação à comparação com o estudo referido acima, no sentido de que os pacientes do presente estudo podem ter mostrado preferência pela frequência mais baixa ao se recordarem da impressão provocada pela frequência muito alta.

A ausência de parâmetros ideais, definidos por estudos clínicos randomizados, demonstra grande inconsistência na área. Dois estudos, realizados com intuito de avaliar a TENS como analgesia em pacientes previamente submetidos à cirurgia de hérnia de disco lombar, usaram diferentes frequências com resultados semelhantes. Um deles encontrou diminuição na dor estabelecendo $80 \mathrm{~Hz}$ como frequência, enquanto outro utilizou $20 \mathrm{~Hz}$ e também evidenciou diminuição clínica da dor, por meio da escala visual analógica de dor. ${ }^{16,17}$

Schulz et al. ${ }^{18}$ afirmam que a contração muscular propiciada pela eletroestimulação de baixa frequência, além de levar à ativação dos mecanismos de liberação dos opioides endógenos, produz um aumento transitório do fluxo sanguíneo arterial para a área estimulada, gerando maior analgesia, sendo eficaz na modulação da dor clínica e experimentalmente induzida. Tal afirmação pode explicar o fato de a agradabilidade relatada pela maioria dos indivíduos ter ocorrido na frequência de $7 \mathrm{~Hz}$, ou seja, baixa frequência. Os supracitados autores citam que a ausência de processos patológicos nos voluntários, com característica amostral semelhante à deste estudo, pode ter influenciado os resultados, uma vez que o enfoque não foi avaliar efeitos analgésicos da corrente, e sim, neste caso, a agradabilidade da mesma. ${ }^{18}$ Como existem dúvidas com relação à frequência e à magnitude da analgesia, é provável que encorajando os pacientes a experimentarem as diversas regulagens de TENS se consiga o resultado mais efetivo. ${ }^{19} \mathrm{Ou}$ seja, podemos entender que nos indivíduos que sentem dor, o fato de esta ceder melhor com as frequências mais altas faz com que não as achem tão desagradáveis.

Conclui-se, de acordo com os resultados obtidos nesta amostragem de indivíduos saudáveis, que tanto o limiar de acomodação quanto o número de acomodações não apresentaram diferenças significativas entre as frequências utilizadas. Em relação à agradabilidade, a frequência de $7 \mathrm{~Hz}$ foi apontada como a mais agradável seguida da frequência de $100 \mathrm{~Hz}$. Uma das limitações deste estudo foi a utilização de apenas três frequências. Pode-se citar ainda, o fato de que os indivíduos da amostra não apresentavam dor, sendo que um dos principais benefícios da TENS é a analgesia. Dessa forma, sugere-se a realização de novos estudos para avaliar a acomodação e a agradabilidade da TENS em amostras de pacientes com algum distúrbio de origem musculoesquelética. 


\section{REFERÊNCIAS}

1. Koca I, Boyaci A, Tutoglu A, Ucar M, Kocaturk O. Assessment of the effectiveness of interferential current therapy and TENS in the management of carpal tunnel syndrome: a randomized controlled study. Rheumatol Int. 2014 Apr 12. [Epub ahead of print].

2. Yu J, Lee S, Kim H, Seo D, Hong J, Lee D. The effect of transcutaneous electrical nerve stimulation on postural sway on fatigued dorsiplantar flexor. Technol Health Care. 2014 Apr 4. [Epub ahead of print].

3. Stein C, Eibel B, Sbruzzi G, Lago PD, Plentz RD. Electrical stimulation and electromagnetic field use in patients with diabetic neuropathy: systematic review and meta-analysis. Braz J Phys Ther. 2013;17(2):93-104.

4. Simpson PM, Fouche PF, Thomas RE, Bendall JC. Transcutaneous electrical nerve stimulation for relieving acute pain in the prehospital setting: a systematic reviewand meta-analysis of randomized-controlled trials. Eur J Emerg Med. 2014;21(1):10-7.

5. Ainsworth L, Budelier K, Clinesmith M, Fiedler A, Landstrom R, Leeper BJ, Moeller L, Mutch S, O’Dell K, Ross J, Radhakrishnan $\mathrm{R}$, Sluka KA. Transcutaneous electrical nerve stimulation (TENS) reduces chronic hyperalgesia induced by muscle inflammation. Pain. 2006;120(1-2):182-7.

6. Pivetta KM, Bertolini GRF. Efeitos do $\Delta \mathrm{F}$ sobre a acomodação da corrente interferencial em sujeitos saudáveis. Rev Bras Med Esporte. 2012;18(5):330-2.

7. Krueger-Beck E, Scheeren EM, Nogueira-Neto GN, Button VLSN, Neves EB, Nohama P. Potencial de ação: do estímulo à adaptação neural. Fisioter Mov. 2010;24(3):535-47.

8. Pallett EJ, Rentowl P, Johnson MI, Watson PJ. Implementation fidelity of self-administered transcutaneous electrical nerve stimulation (TENS) in patients with chronic back pain: an observational study. Clin J Pain. 2014;30(3):224-31.

9. Kılınç M, Livanelioğlu A, Yıldırım SA, Tan E. Effects of transcutaneous electrical nerve stimulation in patients with peripheral and central neuropathic pain. J Rehabil Med. 2014;46(5):454-60.

10. Abreu EA, Santos JDM, Ventura PL. Efetividade da eletroestimulação nervosa transcutânea no alívio da dor durante o trabalho de parto: um ensaio clínico controlado. Rev Dor. 2010;11(4):313-8.

11. Gonçalves RN, Ordenes IEU, Bigaton DR. Efeito indireto da TENS sobre os músculos cervicais em portadores de DTM. Fisioter Mov. 2007;20(2):83-90

12. Lima PMB, Farias RTFB, Carvalho ACA, Silva PNC, Ferraz-Filho NA, Brito RF. Estimulação elétrica nervosa transcutânea após cirurgia de revascularização miocárdica. Rev Bras Cir Cardiovasc. 2011;26(4):591-6.

13. Gondin J, Brocca L, Bellinzona E, D’Antona G, Maffiuletti NA, Miotti D, Pellegrino MA, Bottinelli R. Neuromuscular electrical stimulation training induces atypical adaptations of the human skeletal muscle phenotype: a functional and proteomic analysis. J Appl Physiol (1985). 2011;110(2):433-50.

14. Ferreira J, Beleza ACS. Abordagem fisioterapêutica na dor pós-operatória: a eletroestimulação nervosa transcutânea (ENT). Rev Col Bras Cir. 2007;34(2):127-30.

15. Raimundo AKS, Sousa LA, Silveira RF, Cerqueira MCD, Rodrigues J, Dini PD. Dosagem de serotonina sistêmica após aplicação da eletroestimulação nervosa transcutânea (TENS). Fisioter Mov. 2009;22(3):365-74.

16. Johnston C, Paglioli EB, Paglioli EB. Escore funcional e de dor após cirurgia de hérnia de disco lombar e fisioterapia precoce. Sci Med. 2006;16(4):151-6.

17. Chen W, Yang AT, Dai MT, Fu QL. Observation on therapeutic effect of electroacupuncture under continuous traction for treatment of lumbar discherniation. Zhongguo Zhen Jiu. 2009;29(12):967-9.

18. Schulz AP, Chao BC, Gazola F, Pereira GD, Nakanishi MK, Kunz RI, Canto TO, Carvalho AR, Vilagra JM, Bertolini GRF. Ação da estimulação elétrica nervosa transcutânea sobre o limiar de dor induzido por pressão. Rev Dor. 2011;12(3):231-4.

19. Karakaya IC, Karakaya MG, Erğun E, Elmalı S, Fırat T. Effects of different frequencies of conventional transcutaneous electrical nerve stimulation on pressure pain threshold and tolerance. J Back Musculoskelet Rehabil. 2014;27(2):197-201. 